\title{
Épocas de nacimiento basadas en un índice climático para el ajuste de modelos estadísticos para peso vivo de ganado bovino en México
}

Birth seasons based on a climatic index for statistical model fitting of live weight of bovine cattle in México

Jessica Beatriz Herrera-Ojeda ${ }^{\mathrm{a}}$

Gaspar Manuel Parra-Bracamonte ${ }^{b^{*}}$

Nicolás López-Villalobos ${ }^{\mathrm{c}, \mathrm{d}}$

José Fernando Vázquez-Armijo ${ }^{\mathrm{d}}$

Karlos Edmundo Orozco-Durán ${ }^{e}$

Juan Gabriel Magaña-Monforte ${ }^{\mathrm{f}}$

Juan Carlos Martínez-González ${ }^{\mathrm{g}}$

Francisco Joel Jahuey-Martínez ${ }^{\mathrm{b}}$

a Universidad Michoacana de San Nicolás de Hidalgo-Instituto de Investigaciones Agropecuarias y Forestales. Carretera Morelia-Zinapécuaro km 9.5. 58880. Morelia, Michoacán, México.

${ }^{\mathrm{b}}$ Instituto Politécnico Nacional-Centro de Biotecnología Genómica. Tamaulipas, México.

${ }^{c}$ Institute of Veterinary, Animal and Biomedical Sciences. Massey University. New Zealand.

d Universidad Autónoma del Estado de México. Centro Universitario UAEM Temascaltepec, Estado de México, México.

${ }^{\mathrm{e}}$ Instituto Tecnológico del Valle de Morelia, Tarímbaro, Michoacán. México.

${ }^{\text {f }}$ Universidad Autónoma de Yucatán. Facultad de Medicina Veterinaria y Zootecnia. Yucatán, México. 
g Universidad Autónoma de Tamaulipas. Facultad de Ingeniería y Ciencias. Tamaulipas, México.

*Autor de correspondencia: gparra@ipn.mx

\section{- Resumen:}

El objetivo fue comparar los efectos de una metodología para la clasificación de épocas climáticas, que tradicionalmente son utilizadas para establecer épocas de nacimiento (EN) en estudios y experimentos estadísticos. Esta metodología se basa en un índice de aridez (IA) para clasificar las EN utilizando información meteorológica histórica. Se trabajó una base de datos con un pedigrí de 7,460 animales, se ajustaron dos modelos para peso al nacimiento y peso al destete en bovinos Charolais manejados en pastoreo extensivo. Los modelos incluyeron el efecto fijo de grupo contemporáneo $(\mathrm{GC}=$ subclase de hato, sexo, año y EN) y edad de la madre. Los mismos modelos se compararon utilizando una clasificación tradicional de estaciones del año para clasificar las EN. Al estimar los componentes de varianza y valores genéticos (DEP) con sus exactitudes, los modelos fueron diferentes de acuerdo a la prueba de razón de verosimilitudes $(P<0.01)$. El número de GC se redujo en $25 \%$ para la EN basada en el IA, con GC con mayor número de individuos. El principal efecto observado en los modelos analizados fue el cambio en la jerarquización de las DEP para ambas características. Este método de clasificación de épocas de nacimiento, puede ayudar a mejorar el ajuste de modelos estadísticos en los sistemas ganaderos manejados en pastoreo extensivo.

- Palabras clave: Bovinos, Charolais, Evaluación genética, Indice de aridez, Modelos, Peso vivo.

\section{- Abstract:}

The objective of this study was to compare the effects of a methodology for the climatic season classification that traditionally had been used as birth season (BS) in statistical assessments. This methodology bases in an aridity index (AI) to classify BS using meteorological historic information. Using a 7,460-pedigree animals dataset were fitted two statistical models for birth and weaning weights of Charolais cattle. Genetic models included the fixed effect of contemporary groups ( $\mathrm{CG}=$ herd, sex, year and $\mathrm{BS})$ and dam age as a covariate (linear and quadratic). Same models compared with a traditional season classification for BS. When variance component and expected progeny differences (EPD) 
were predicted with their accuracies, models were statistically different accordingly to the likelihood ratio test $(P<0.01)$. An improvement and reduction in CG conformation $(25 \%)$ were observed for those models including IA based BS. The main effect in assessed models was the ranking changes in EPD from both traits. This classification method of birth season can improve the model fitting in animal production based in extensive systems.

- Palabras clave: Aridity index, Cattle, Charolais, Genetic evaluation, Live weight.

Recibido 13/06/2017

Aceptado 03/01/2018

\section{Introducción}

El clima puede afectar el desempeño del ganado de diferentes maneras; modifica la calidad o cantidad de los alimentos disponibles, el requerimiento de agua y energía, el consumo energético y su uso. El efecto del clima en la producción animal se reconoce como un factor altamente variable y complejo que condiciona e impacta el ambiente en el que los animales viven, se desempeñan y se reproducen ${ }^{(1,2)}$. El ganado enfrenta los efectos del clima mediante diferentes mecanismos de modificación de las constantes fisiológicas y etológicas; de esta manera mantiene su homeostasis en respuesta a las condiciones ambientales $^{(3)}$. Como consecuencia, es posible observar alteraciones en el consumo de materia seca, en el comportamiento, actividad física y productividad, que se reflejan en cambios en algunos indicadores económicos, como el peso al nacimiento, producción lechera, peso al destete y ganancia diaria de peso. Estos efectos impactan significativamente los sistemas productivos basados en condiciones extensivas de manejo ${ }^{(4,5,6)}$.

Es común que al utilizar modelos estadísticos para evaluar la productividad animal y sus componentes, uno de los principales factores a considerar sean las épocas climáticas. Los diferentes criterios para establecer dichas épocas contemplan generalmente componentes climáticos que son inexactos al explicar las variaciones que definen un determinado periodo del año, ya que el clima está determinado por circunstancias atmosféricas globales, pero posee un comportamiento específico para cada zona o región ${ }^{(7)}$. En ganado bovino en 
sistemas extensivos de pastoreo existe una compleja interacción de factores como la temperatura, humedad relativa, viento, precipitación y radiación solar, siendo éstas las principales variables que influyen en la productividad ${ }^{(8)}$. A la fecha, no existe una metodología que incluya dichos elementos como condicionantes de la variación del tiempo atmosférico en una época particular del año, y menos aún, relacionados con indicadores de crecimiento en bovinos. Por ello se propone una metodología más eficiente y conveniente para clasificar y describir períodos climáticos en una zona determinada de estudio. El propósito es aportar información que minimice las fuentes de variación que inciden sobre la expresión fenotípica de los indicadores productivos y poder mejorar la estimación de los valores y parámetros requeridos en la evaluación genética en los hatos y a nivel individual.

Los modelos usados para análisis estadísticos de características productivas incluyen la época o el mes de nacimiento como uno de los principales factores que inciden sobre la expresión del potencial productivo de los animales ${ }^{(9,10)}$. En las evaluaciones genéticas, para poder estimar parámetros y valores de cada animal con alta confiabilidad, los modelos incluyen la época de nacimiento como un efecto fijo dentro de las ecuaciones de los modelos mixtos, lo que ayuda a aislar mejor los componentes de la varianza de los efectos aleatorios, lo que se ha considerado como el mejor estimador lineal insesgado ${ }^{(11)}$. El mes o época de nacimiento (periodo estacional en el que ocurre el nacimiento), puede llegar a contribuir hasta con el $13 \%$ de la variación de los pesos al nacimiento (PN) y al destete $(\mathrm{PD})^{(12)}$. La época de nacimiento involucra diversos factores climáticos reconocidos por impactar la variabilidad de estos indicadores de producción ${ }^{(5)}$; generalmente los efectos ambientales que determinan la variación en las características de crecimiento son diferentes según la zona agroecológica, el sistema de producción y la población que se estudia ${ }^{(13,14)}$. Cuando se han tratado de definir estos períodos climáticos para el ajuste de modelos estadísticos en características productivas, el criterio principal para la clasificación es la definición específica asociada a la presencia o ausencia de la precipitación pluvial, clasificando los climas simplemente en secos o lluviosos ${ }^{(15,16)}$, aunque algunos autores han considerado también la temperatura ${ }^{(17)}$, estaciones del año ${ }^{(17,18)}$ o criterios definidos por clústeres entre meses durante el año ${ }^{(19,20)}$.

El objetivo de este estudio fue evaluar los efectos de un método matemático para clasificar períodos climáticos considerando el índice de aridez en diferentes regiones de estudio, sobre parámetros productivos y estimaciones en el ajuste de modelos estadísticos de evaluación genética en ganado bovino criado en condiciones extensivas. 


\section{U Material y métodos \|}

\section{- Información climática •}

La información climatológica utilizada se obtuvo por medio del Extractor Rápido de Información Diaria (ERIC III versión 3.2.), que facilita la obtención de información de la base de datos CLICOM (clima computarizado) del banco de datos histórico de la Comisión Nacional del Agua ${ }^{(21)}$. La información utilizada fue de datos históricos de 30 años (1985 a 2015) de información meteorológica diaria de la precipitación pluvial (PP), temperaturas medias (TM), mínimas y máximas, radiación solar (RS), humedad (H) y velocidad del viento (VV). La clasificación propuesta se logró a través de la elaboración de un índice de aridez (IA), utilizando la precipitación pluvial y el índice de evapotranspiración (ET0). Bajo un método descrito previamente ${ }^{(22)}$ como:

$$
E T 0=2.45 \times 0.1555 \times \operatorname{Rax} \sqrt{T_{\text {Max }}+T_{\text {Min }}} \times\left(9.96710^{-2}+4.28010^{-3} \frac{T_{\text {Max }}+T_{\text {Min }}}{2}\right)
$$

\section{Donde:}

ET0= Índice de evapotranspiración,

$\mathbf{R a}=$ Radiación solar extraterrestre,

TMax $_{\text {Memperatura máxima, }}$

$\mathbf{T}_{\text {Min }}=$ Temperatura mínima.

Posteriormente, se estimaron los IA en cada una de las estaciones, por medio de la metodología propuesta en el Diario Oficial de la Federación (1 de junio de 1995), para desarrollar criterio clasificatorio de la aridez a partir de la relación entre la precipitación pluvial diaria (PP) y la ET0, lo que permite clasificar regiones en áridas, semiáridas, subhúmedas y húmedas ${ }^{(23)}$, considerando los niveles de 0.0 a 0.20 para clasificar un mes árido (A), 0.21 a 0.50 como semiárido (SA), 0.51 a 0.65 como subhúmedo (SH) y $>0.65$ como húmedo (H) (Cuadro 1). De esta manera la dinámica de IA fue considerada para cada hato particular de acuerdo a las estimaciones calculadas (Figura 1). Las ecuaciones para elaborar el ET0 se estimaron en el software $\mathrm{R}^{(24)}$. 
Cuadro 1: Categorías del índice de aridez (IA) utilizadas para la reclasificación de las épocas climáticas

\begin{tabular}{lcc}
\hline Escala de IA & Categorías & Clave \\
\hline 0.00 a 0.20 & Áridos & $\mathrm{A}$ \\
0.21 a 0.50 & Semiáridos & SA \\
0.51 a 0.65 & Subhúmedos secos & SH \\
$>0.65$ & Húmedos & H \\
\hline
\end{tabular}

Figura 1. Tendencias anuales de índice de aridez en tres localidades estudiadas

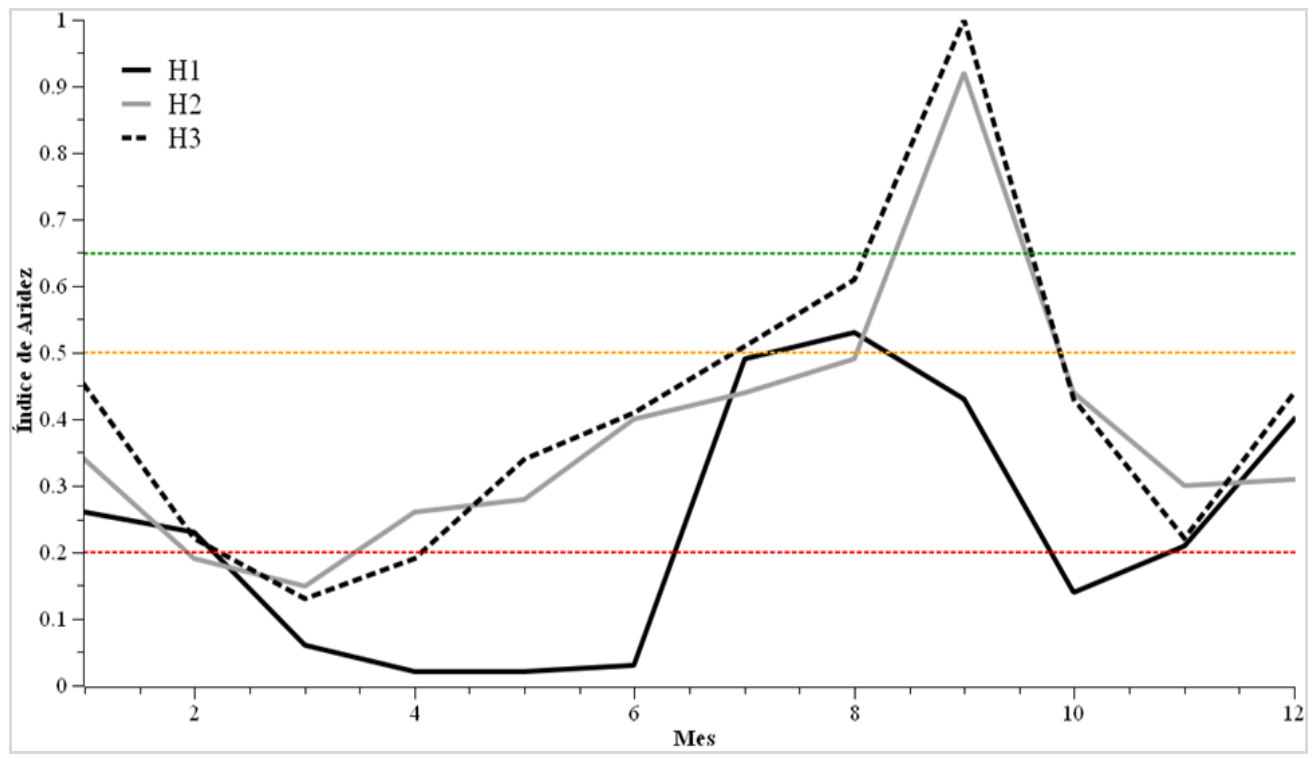

Líneas punteadas (roja, naranja y verde): límite de clasificación de época de acuerdo al Cuadro 1. (H1= Hermosillo, H2= Mamulique, H3= Salinas Victoria).

\section{- Efecto de la clasificación propuesta •}

Se utilizaron las bases de datos de tres hatos de ganaderías de registro de ganado Charolais, en Sonora y Nuevo León, México, que contaban con información genealógica y con registros de producción. Conformando un pedigrí de 7,460 animales. Utilizando la localización geográfica de cada hato se obtuvo la información meteorológica diaria de tres estaciones cercanas a las regiones de los hatos incluidos en el estudio. Las estaciones 
meteorológicas consideradas fueron Hermosillo, Sonora (H1: 2905'44"'N, 110 57'03”O),

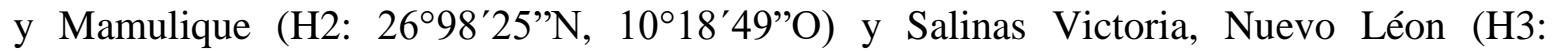
$\left.25^{\circ} 58^{\prime} 00^{\prime \prime}\right)$.

Una vez establecidos los períodos climáticos por localización geográfica, utilizando el criterio de IA, se compararon dos modelos de ajuste para analizar las variables dependientes. Como comparación se consideró una clasificación previamente descrita ${ }^{(20)}$, donde se consideraron las épocas de nacimiento en: 1) enero-marzo; 2) abril-junio; 3) julioseptiembre y 4) octubre-diciembre, en un análisis de parámetros genéticos con ganado Charolais, de tal manera que el primer modelo se definió de acuerdo a esta clasificación para representar el método tradicional (MTR). El segundo modelo considerado en el estudio fue la propuesta con el criterio de IA (MIA). Se examinó la correlación entre las dos clasificaciones comparadas mediante el coeficiente de correlación de Spearman, utilizando el paquete estadístico SAS ${ }^{(25)}$.

Para examinar la proporción de varianza explicada por la época de nacimiento (EN) en cada uno de los métodos (MTR y MIA) sobre el peso al nacimiento (PN) y peso al destete (PD), se ajustó un modelo mixto que consideró los efectos fijos de hato, el sexo del animal, y los efectos lineal y cuadrático de la edad de la vaca como covariable, más el efecto aleatorio de la EN, del semental y del año de nacimiento. Estos análisis se realizaron utilizando el PROC MIXED del paquete estadístico SAS ${ }^{(25)}$.

Posteriormente, se ajustaron los mismos modelos (MTR y MIA), para estimar los componentes de varianza bajo el método de máxima verosimilitud restringida libre de derivadas en el paquete de evaluaciones genéticas MTRDFREML ${ }^{(26)}$, el modelo para PN y PD fue, en forma matricial:

$$
Y=X \beta+Z d+W m+e
$$

\section{Donde:}

$\boldsymbol{Y}$ es el vector de observaciones para $\mathrm{PD}, X, Z$ y $W$ con matrices conocidas de incidencia que relacionan las observaciones con sus respectivos vectores de efectos fijos y aleatorios;

$\boldsymbol{\beta}$ es el vector de efectos fijos antes descritos;

$\boldsymbol{d}$ es el vector de efectos genéticos aditivos directos;

$\boldsymbol{m}$ es el vector de efectos genéticos aditivos maternos;

$\boldsymbol{e}$ es el vector de efectos aleatorios residuales.

En ambos modelos el grupo contemporáneo estuvo integrado por animales del mismo hato, sexo, año y época de nacimiento. La época de nacimiento se diferenció por la reclasificación entre modelos. El criterio de convergencia de los modelos se fijó en 1×10-19. 
Para la comparación de modelos se utilizó una prueba de razón de verosimilitudes, que se basa en las diferencias entre los logaritmos de las funciones de verosimilitud (Log L), estimadas en el programa y comparadas con el valor de Ji-cuadradra $\left(\mathrm{X}^{2}\right)$ con un grado de libertad con un alpha $=0.01$. La prueba de acuerdo a Lynch y Walsh ${ }^{(27)}$ puede presentarse como $\lambda=-2(\log$ L MIA - Log L MTR). Donde Log L= logaritmo de verosimilitud, con las reglas de decisión: Si $\lambda \geq x^{2}$, el modelo MIA se acepta significativamente como diferente al modelo MTR y si $\lambda \leq \mathrm{x}^{2}$, el modelo MTR y el MIA no son significativamente diferentes.

De cada modelo se estimaron las diferencias esperadas en la progenie (DEP) y sus exactitudes, estimando sus medias y desviaciones típicas. Posteriormente se extrajo el $10 \%$ superior de los valores de DEP y sus exactitudes de acuerdo a una jerarquización basada en el modelo MTR para cada característica, y considerando para PN los valores inferiores y para el PD los valores superiores. Se estimó la correlación de rango de Spearman entre estos valores predichos en cada modelo por característica para determinar cambios en el ordenamiento de los animales superiores, usando el procedimiento CORR del paquete estadístico SAS v $9.2^{(25)}$

\section{Resultados y discusión}

En el presente estudio las dos características de peso vivo fueron similares a las reportadas previamente en ganado Charolais mexicano en sistemas extensivos ${ }^{(20,22)}$ con 39.0 y 227.0 kg para PN y PD, respectivamente (Cuadro 2).

Cuadro 2: Estadísticos descriptivos para peso al nacimiento (PN) y peso al destete ajustado a 205 días (PD) de bovinos Charolais de registro $(\mathrm{kg})$

\begin{tabular}{lcccccc}
\hline Característica & n & Media & SD & CV & Máximo & Mínimo \\
\hline PN & 6398 & 40.51 & 7.36 & 18.18 & 66.00 & 15.00 \\
PD & 5901 & 228.52 & 41.76 & 18.28 & 396.60 & 101.50 \\
\hline
\end{tabular}

$\mathrm{n}=$ número de observaciones; $\mathrm{DE}=$ desviación estándar; $\mathrm{CV}=$ coeficiente de variación.

Recientemente, se propuso un método para reclasificar épocas climáticas basadas en un índice de aridez, estimando el efecto de este cambio en el análisis de modelos mixtos de 
evaluación genética con una pequeña población de ganado Charolais ${ }^{(22)}$. En el presente trabajo se comparó el método propuesto con una población conformada por un pedigrí con mayor número de animales.

No es frecuente encontrar estudios que evalúen la importancia específica del componente ambiental en el ajuste de modelos estadísticos como los de evaluación genética. La mayoría da por hecho que la inclusión de los factores que son importantes como fuentes de variación puede basarse en criterios que se usan de manera general (por ejemplo, la conformación de grupos contemporáneos) y cuando no exista un problema con la estructura de los datos, o con la elección de los niveles que conforma el vector de los efectos fijos (por ejemplo, el sexo) ${ }^{(28)}$, lo que consecuentemente conformará el BLUE (Mejor Estimador Lineal no Sesgado; por sus siglas en inglés $)^{(11)}$. Para el caso de factores que explican una proporción sustancial de la variación fenotípica y poseen complejidad para su conformación, es necesario considerar su preclasificación de acuerdo a un criterio objetivo para seleccionar su inclusión, ya sea individualmente o en bloques, como los grupos contemporáneos, sobre todo en el caso de evaluaciones genéticas que incluyen diferentes sistemas de producción con diferencias agroclimáticas.

Para el establecimiento de épocas de nacimiento que conforman los grupos contemporáneos en evaluaciones genéticas, una clasificación provista por el IA explica sustancialmente mayor variabilidad que una clasificación tradicional, por lo que la IA es capaz de aislar y explicar en algunos casos aproximadamente el $10 \%$ de la variabilidad de la característica, lo que indica un mejoramiento en la estructura de los factores analizados y el número de observaciones que contiene ${ }^{(22)}$.

Los cambios observados en la varianza genética aditiva para PN en los componentes de la varianza encontrados en el presente estudio fueron pequeños (Cuadro 3). Al comparar la heredabilidad directa obtenida en MIA contra MTR se encontraron cambios menores entre modelos; de la misma forma para PD el ajuste del modelo MIA, mostró valores ligeramente superiores en sus componentes de la varianza, sobre todo en la varianza genética aditiva y la covarianza entre la varianza genética directa y materna, excepto en la varianza materna, en la cual hubo una ligera reducción para el PD. En ambas comparaciones (PN y PD) los modelos fueron significativamente diferentes de acuerdo a la prueba de razón de verosimilitudes $(P<0.01)$, lo que sugiere que la inclusión del IA produce un cambio en la estructura de los datos que influyen en la captura de la varianza genética aditiva estimada y subsecuentemente en la predicción genética. 
Cuadro 3: Componentes de la varianza y parámetros genéticos a partir del ajuste de dos modelos para caracteres de peso vivo en ganado Charolais manejado en condiciones extensivas

\begin{tabular}{lcccc}
\hline \multirow{2}{*}{$\begin{array}{c}\text { Componentes } \\
\text { y parámetros }\end{array}$} & MTR & MIA & MTR & MIA \\
\cline { 2 - 5 } & 13.276 & 15.103 & 265.989 & 341.904 \\
$\boldsymbol{\sigma}_{d}^{2}$ & 11.864 & 12.571 & 234.165 & 269.442 \\
$\boldsymbol{\sigma}_{\boldsymbol{m}}^{\mathbf{2}}$ & -7.912 & -8.810 & -67.543 & -109.800 \\
$\boldsymbol{\sigma}_{\boldsymbol{d m}}$ & 21.219 & 21.292 & 595.888 & 652.066 \\
$\boldsymbol{\sigma}_{\boldsymbol{e}}^{\mathbf{2}}$ & 38.448 & 40.156 & 1028.888 & 1153.613 \\
$\boldsymbol{\sigma}_{\boldsymbol{p}}^{\boldsymbol{2}}$ & $28091.6333^{\mathrm{a}}$ & $28537.0584^{\mathrm{b}}$ & $44068.0588^{\mathrm{a}}$ & $45238.2424^{\mathrm{b}}$ \\
$-2 \log \mathrm{L}$ & $0.35 \pm 0.05$ & $0.38 \pm 0.05$ & $0.26 \pm 0.04$ & $0.30 \pm 0.04$ \\
$\boldsymbol{h}^{\mathbf{b}}$ & $0.31 \pm 0.03$ & $0.31 \pm 0.03$ & $0.24 \pm 0.03$ & $0.23 \pm 0.03$ \\
$\boldsymbol{m}^{2}$ & $-0.63 \pm 0.05$ & $-0.64 \pm 0.05$ & $-0.27 \pm 0.10$ & $-0.36 \pm 0.09$ \\
$\boldsymbol{r}_{\boldsymbol{d m}}$ & $0.55 \pm 0.03$ & $0.53 \pm 0.03$ & $0.58 \pm 0.03$ & $0.57 \pm 0.03$ \\
$\boldsymbol{e}^{\mathbf{2}}$ &
\end{tabular}

MTR= modelo de época tradicional, MIA= modelo con la época de acuerdo al IA (Cuadro 1). $\boldsymbol{\sigma}_{d}^{2}=$ Varianza genética directa; $\boldsymbol{\sigma}_{m}^{2}=$ Varianza genética materna; $\boldsymbol{\sigma}_{d m}=$ Covarianza genética directa y materna, $\boldsymbol{\sigma}_{e}^{2}=$ Varianza ambiental, $\boldsymbol{\sigma}_{p}^{2}=$ Varianza fenotípica; $\mathrm{h}^{2}=$ heredabilidad directa; $\mathrm{m}^{2}=$ heredabilidad materna; $\mathrm{r}_{\mathrm{dm}}=$ correlación directa-materna, $\mathrm{e}^{2}=$ proporción de varianza ambiental relativa a la varianza fenotípica; $\log \mathrm{L}=\log$ aritmo de verosimilitud. a,b Literales distintas entre modelos en cada característica son diferentes $(P<0.01)$.

Estos resultados demuestran que la reclasificación de la EN, probablemente tenga mayores efectos en el ajuste de modelos más complejos, que incluyan además del efecto genético directo, el efecto materno debido a las conexiones genéticas que puedan establecerse entre los grupos contemporáneos, y con poblaciones con mayor número de evaluaciones nacionales, en las que sean incluidas más regiones del país en condiciones climáticas heterogéneas. Un aspecto importante entre los datos analizados de cada modelo, es que MIA mostró una reducción muy importante $(>25 \%)$ en el número de los grupos contemporáneos en la base de datos analizada, lo que pudiera sugerir grupos de comparación más grandes y mejor distribuidos por año. Se ha propuesto que este aspecto es fundamental en las evaluaciones genéticas, ya que la predicción de valores genéticos depende principalmente de la correcta estimación de los componentes de varianza; así como de la correcta definición de los efectos medioambientales. Una mejor definición de 
los efectos fijos responsables de la variación fenotípica puede ayudar a disminuir sesgos causados por diferencias en las condiciones ambientales de los animales ${ }^{(29,30)}$.

Los grupos contemporáneos en las evaluaciones genéticas han sido una estrategia fundamental para lograr reducir la variabilidad causada por diferentes efectos ambientales. Dentro de tal estrategia resulta clave la búsqueda de una mejor definición de los grupos contemporáneos, en cuya clasificación se incluyan factores que permitan predicciones más reales del mérito genético en indicadores de interés económico. Esto es esencial en el proceso de selección para ganado de carne, ya que de esto depende en parte el progreso genético de la población ${ }^{(31,32)}$.

El análisis específico de los promedios de los valores predichos en las DEP y sus exactitudes (Cuadro 4) no sugiere cambios sustantivos en la distribución poblacional de los predictores genéticos o de sus valores de confiabilidad. Los promedios y sus desviaciones típicas son similares entre modelos para las dos características de peso vivo estudiadas. Sin embargo, al analizar el $10 \%$ de los animales superiores clasificados con base en las DEP, la estimación de los coeficientes de correlación, mostró que el efecto en los valores predichos que clasifican a los individuos incluidos en la evaluación genética es importante (Cuadro 5). Los menores valores de correlación se observaron entre las DEP directas de PN y PD. La reducción de la categorización de las DEP también fue notoria en los predictores maternos.

Cuadro 4: Medias y desviaciones típicas de las diferencias esperadas en la progenie y exactitudes en dos modelos evaluados en caracteres de peso vivo de ganado Charolais

\begin{tabular}{lcccc}
\hline \multirow{2}{*}{$\begin{array}{l}\text { Valor } \\
\text { predicho }\end{array}$} & \multicolumn{2}{c}{ PN } & \multicolumn{2}{c}{ PD } \\
\cline { 2 - 5 } & MTR & MIA & MTR & MIA \\
\hline DEP D & $-0.160 \pm 1.920$ & $-0.144 \pm 2.132$ & $2.151 \pm 9.068$ & $2.247 \pm 10.302$ \\
EX D & $0.529 \pm 0.168$ & $0.546 \pm 0.175$ & $0.519 \pm 0.140$ & $0.535 \pm 0.154$ \\
DEP M & $0.408 \pm 1.864$ & $0.391 \pm 1.935$ & $1.302 \pm 7.445$ & $1.197 \pm 7.585$ \\
EX M & $0.535 \pm 0.104$ & $0.541 \pm 0.102$ & $0.495 \pm 0.118$ & $0.492 \pm 0.114$ \\
\hline
\end{tabular}

MTR= modelo de época tradicional, MIA= modelo con la época de acuerdo al IA (Cuadro 1), DEP D= diferencias esperadas de las progenies directas; EX D= exactitudes de la DEP D; DEP M= diferencias esperadas de las progenies maternas; EX M= exactitud de la DEP M. 
Cuadro 5: Coeficientes de correlación entre las diferencias esperadas de la progenie y sus exactitudes de caracteres de peso vivo de ganado Charolais, estimados con dos modelos

\begin{tabular}{llc}
\hline \multirow{2}{*}{ Característica } & \multicolumn{2}{c}{ Correlación de Spearman } \\
\cline { 2 - 3 } & DEP & Exactitud \\
\hline PN D & 0.646 & 0.96 \\
PN M & 0.895 & 0.99 \\
PD D & 0.736 & 0.97 \\
PD M & 0.954 & 0.99 \\
\hline
\end{tabular}

$\mathrm{DEP} D=$ diferencias esperadas de las progenies directas; PN D y PD $\mathrm{D}=$ diferencias esperadas de las progenies directas para peso al nacimiento y destete, respectivamente; PN M y PD M= diferencias esperadas de las progenies maternas para peso al nacimiento y destete, respectivamente.

Los cambios identificados entre los modelos estudiados podrían explicarse por el cambio en la estructura de los grupos contemporáneos ocasionada por la reclasificación de la época de nacimiento. Algunas de las aproximaciones utilizadas en las publicaciones disponibles nacionalmente no proveen evidencia sustancial sobre sus criterios de clasificación. Las épocas de nacimiento entre los diversos estudios realizados en México se definen constantemente considerando dos variables climatológicas: la distribución del patrón de precipitación y la temperatura mensual de acuerdo a un promedio histórico. En este sentido, existen reportes donde para evaluar la magnitud de interacción entre genotipos de ganado Suizo Europeo y el clima se clasificaron grupos contemporáneos con épocas de nacimiento definidas por la presencia de lluvias a través de los años estudiados y temperaturas mensuales (secas: junio-octubre y lluvias: noviembre-mayo) ${ }^{(33)}$; aunque para evaluar la interacción genotipo-ambiente en este estudio se definieron clústeres con zonas climatológicas para México (trópico seco: oeste y sureste; trópico húmedo: este y templado: norte y centro), éstas no se tomaron en cuenta para una mejor clasificación de las épocas de nacimiento. De igual manera, al explorar el efecto de los GC de acuerdo a la cantidad de registros que tuvieran (3, 7 o 10 registros) y su inclusión como un efecto fijo o aleatorio, algunos autores establecieron grupos contemporáneos de acuerdo a la distribución de las lluvias y la temperatura registrada por la estación meteorológica más cercana a cada unidad de estudio ${ }^{(13)}$.

Por otra parte, se ha utilizado la presencia de la precipitación y las temperaturas mensuales para establecer épocas (secas, lluvias y nortes) en cinco Estados del sureste y del norte de México (Campeche, Tabasco, Chiapas, Tamaulipas y Yucatán), justificando que estos Estados comparten un clima similar (caliente subhúmedo con lluvias en verano) ${ }^{(17)}$. Sin 
embargo, de acuerdo a los resultados de este estudio, cuando se utilizan otras variables climatológicas, los estados del norte mostraron comportamiento diferente a los del sureste del país.

Algunos autores tratando de definir el modelo estadístico más apropiado para la evaluación genética de indicadores de crecimiento en ganado mexicano, escogieron utilizar como único criterio para las épocas de nacimiento la presencia de lluvia, sin especificar la fuente de información utilizada para categorizar esta variable ${ }^{(28,34,35)}$. Las condiciones particulares de la clasificación de grupos contemporáneos reportados en la literatura no incluyen dentro de la estructura de los datos, elementos climatológicos importantes, que en este estudio han demostrado acercar los modelos estadísticos a una situación más real para los datos.

Aunque estos ligeros cambios en los parámetros genéticos y la diferencia encontrada en el ordenamiento de los valores genéticos diferencialmente podrían significar un mejor ajuste en el modelo con IA como criterio de clasificación de la EN, la superioridad de sus efectos solamente se podrían confirmar si se considerara el análisis incluyendo el valor genético verdadero de una población de animales.

\section{- Clasificación de épocas climáticas •}

En la Figura 1, se observan las curvas de IA estimadas para las tres localidades de los hatos incluidas en el estudio. Las tendencias observadas en el gráfico indican que las condiciones climáticas son similares desde junio hasta noviembre para $\mathrm{H} 2$ y H3; estos meses se ubican entre subhúmedos y húmedos por estar localizados en una región relativamente cercana (menos de $200 \mathrm{~km}$ de distancia), pero muy diferentes a H1 en el cual se observó que el único mes subhúmedo es agosto.

En países como México donde los sistemas de producción de ganado de carne se basan en áreas de pastoreo y agostaderos, es común que los animales se expongan a los efectos medioambientales en distinta magnitud, debido a diferencias en la variedad y calidad del forraje, topografía y a las variables climáticas. Los componentes del clima (radiación solar, humedad relativa, precipitación, temperatura ambiental, velocidad del viento) en conjunto ejercen una acción directa en la industria ganadera, afectando el grado de bienestar fisiológico de los animales y un efecto indirecto a través de la producción y calidad de los alimentos, documentándose su influencia en la eficiencia de los sistemas de producción de bovinos de carne en regiones con clima tropical ${ }^{(1,36)}$. 
Para poder capturar las variables del clima que pueden afectar tanto al animal como a la oferta de alimento en los sistemas de pastoreo extensivo, el índice de aridez, permite evaluar el agua que se pierde tanto por evaporación como por la transpiración de los forrajes, y al mismo tiempo incluyendo variables fisiológicas que en el ganado se traducen en cambios de temperatura corporal, tasa de respiración y la sudoración, así como el consumo de agua y materia seca ${ }^{(37)}$. Por lo tanto, el uso de este criterio considera de manera más efectiva las condiciones a las que los animales o cultivos forrajeros se someten en condiciones reales, lo que ayudaría a remover o estimar la variación que explica fenotípicamente los caracteres que se expresan bajo condiciones extensivas. Bajo esta premisa, las épocas climáticas estimadas con la metodología propuesta serían equivalentes a pesar de la región geográfica, dado que los componentes climáticos considerados en su clasificación serían comparables, lo que podría explicar el por qué en algunas regiones se consideraría la existencia de dos épocas climáticas, cuando en otras podrían existir las cuatro, independientemente del mes.

En el presente trabajo, la estimación de índices de aridez para 106 municipios en diferentes Estados de México está disponible en el Anexo 1 para fines de análisis. Estos valores podrían ser utilizados como referencia para clasificación de las épocas en estudios que incluyan experimentos o datos de campo bajo sistemas productivos en condiciones extensivas.

\section{" Conclusiones e implicaciones \|}

El estudio realizado utilizando un mayor número de animales en una base de datos con un mayor pedigrí, permitió comprobar que el índice de aridez definido como criterio de clasificación de meses y épocas de nacimiento para incluir los factores ambientales que inciden sobre el desempeño productivo de los animales en condiciones extensivas es útil. El análisis entre los modelos de evaluación genética incluyendo el contraste de grupos contemporáneos en el índice de aridez mostró que su implementación no produce cambios específicos en los parámetros genéticos, pero sí en el ordenamiento de los individuos basados en sus valores genéticos aditivos predichos. Esta información podría ayudar a hacer más precisos los programas de mejora genética al analizar modelos complejos. Con base en los resultados, el uso del índice de aridez puede extenderse a diferentes esquemas de aplicación agropecuaria en los cuales animales o cultivos estén sometidos a condiciones 
extensivas cuando se tenga disponibilidad de la información climatológica que permita esta clasificación.

\section{- Literatura citada:}

1. Arias RA, Mader TL, Escobar C. Factores climáticos que afectan el desempeño productivo del ganado bovino de carne y leche. Arch Med Vet 2008;(40):7-22.

2. Berman A. Are adaptations present to support dairy cattle productivity in warm climates? J Dairy Sci 2011;(94):2147-2158.

3. Wheelock JB, Rhoads RP, VanBaale MJ, Sanders SR, Baumgard LH. Effects of heat stress on energetic metabolism in lactating Holstein cows. J Dairy Sci 2009;(93):644655.

4. Nardone A, Ronchi B, Lacetera N, Bernabucci U. Climatic effects on productive traits in livestock. Vet Res Commun 2006;(30):75-81.

5. Nienaber JA, Hahn GI. Livestock production system management responses to thermal challenges. Int J Biometeorol 2007;(52):149-157.

6. Tao S, Bubolz JW, Amaral BC, Thompson IM, Hayen MJ, Johnson SE, Dahl GE. Effect of heat stress during the dry period on mammary gland development. J Dairy Sci 2011;(94):5976-598.

7. Morillo DE. Efectos de la época seca sobre la producción forrajera y bovina. Rev Fac Agron 1994;(15):2-7.

8. Hahn GL, Mader TI, Eigenberg RA. Perspective on development of thermal indices for animal studies and management. In: Lacetera N, et al editors. Interactions between climate and animal production. 1rst ed. Wageningen, The Netherlands: Wageningen Academic Publishers; 2003:31044-31056.

9. Martínez JCM, Martínez AA, Hernández J, Parra GM, Castillo SP. Características predestete de bovinos Simmental (Bos taurus) y sus cruces con Brahman (Bos indicus) en el trópico mexicano. Rev Colomb Cienc Pecu 2008;(21):365-371.

10. Chin RC, Magaña JG, Segura JC, Núñez R. Factores ambientales y proporción racial que influyen en el peso al nacimiento, al destete y edad al primer parto en bovinos Suizo Pardo en México. Trop Sub-trop Agroeco 2012;(15):55-60.

11. Mrode RA. Linear models for the prediction of animal breeding values. 3rst ed. Oxxford, United Kingdom: Cabi International; 2014. 
12. Martínez GG, Petrocinio J, Herrera PD. Factores que afectan el peso al destete en un rebaño de bovinos de carne. Rev Fac Agron 1998;(15):2-7.

13. Ramírez-Valverde R, Nuñez-Domínguez R, Ruíz-Flores A, García-Muñíz G, MagañaValencia F. Comparación de definiciones de grupos contemporáneos en la evaluación genética de bovinos suizo europeo. Téc Pecu Méx 2008;46(4):359-370.

14. Garrick DJ. An animal breeding approach to the estimation of genetic and environmental trends from field populations. J Anim Sci 2010;(88):E3-E10.

15. Parra-Bracamonte GM, Martínez JC, García FJ; González AM, Briones F, Cienfuegos EG. Tendencias genéticas y fenotípicas de características en el ganado Brahaman de registro de México. Rev Cient 2007;(17):262-267.

16. Martínez-González JCM, García-Esquivel FJ, Parra-Bracamonte GM, Castillo-Juárez H, Cienfuegos-Rivas EG. Genetic parameters for growth traits in mexican Nellore cattle. Trop Anim Health Prod 2010;(42):887-892.

17. Medina-Zaldívar JM, Osorio-Arce MM, Segura-Correa JC. Influencias ambientales y parámetros genéticos de crecimiento en ganado Nelore en México. Rev Cient FCVLuz 2005;(3):325-241.

18. Martínez-González JC, Gutiérrez-Michel JF, Briones-Encinia F, Lucero-Magaña FA, Castillo-Rodríguez SP. Factores no genéticos que afectan el peso al nacer y destete de terneros Angus. Zootecnia Trop 2011;(2):151-159.

19. Ríos A, Velázquez GM, Tsuruta S, Bertrand JK, Murillo VEV, Bermúdez MM. Estimadores de parámetros genéticos para características de crecimiento de ganado Charolais mexicano. Téc Pecu Méx 2007;45(2):121-130.

20. Ríos UA, Martínez G, Vega VG, Montaño M. Efectos genéticos para características de crecimiento de bovinos Charolais y Charbray mexicanos estimados con modelos alternativos. Rev Mex Cienc Pecu 2012;3(3):275-290.

21. CONAGUA. 2016. Banco de datos histórico. Comisión Nacional del Agua. http://sMIA.cna.gob.mx/informacion-climatologica-ver-estado?estado=son. Consultado 30 May, 2017.

22. Herrera-Ojeda JB, Parra-Bracamonte GM, Herrera-Camacho J, López-Villalobos N, Magaña-Monforte JG, Martínez-González JC, Lobit P, Vázquez-Armijo JF. Información climática asociada a estaciones productivas para el ajuste de modelos estadísticos de sistemas bovinos bajo condiciones extensivas. Arch Zootec 2018;(257):21-28. 
23. UNEP. United Nations Environment Programme. Biodiversity Country Studies: Executive Summary. New York: UNEP. 1992.

24. R Development Core Team R: A language and environment for statistical computing. R Foundation for Statistical Computing, Vienna Austria. 2008 http://www.Rproject.org.

25. SAS Institute. SAS/STAT Software: Changes and enhancements through Release 9.1 Cary, NC. 1996.

26. Boldman KG, Kriese LA, VanVleck LD, VanTassell CP, Kachman SD. A manual for use of MTDFREML. A set of programs to obtain estimates of variances and covariances [DRAFT]. USDA Agr Res Serv. USA. 1995.

27. Lynch M, Walsh B. Genetics and analysis of quantitative traits 1rst ed. Sunderland. USA; Sinauer; 1998.

28. Wilson AJ, Reale D, Clements MN, Morrissey MM, Postma E, Walling CA, Kruuk LE, Nussey DH. An ecologist's guide to the animal model. J Anim Ecol 2010;(79):1326.

29. Domínguez-Viveros J, Rodríguez-Almeida FA, Ortega-Gutiérrez JA, FloresMariñelarena A. Selección de modelos, parámetros genéticos y tendencias genéticas en las evaluaciones genéticas nacionales de bovinos Brangus y Salers. Agrociencia 2009;(43):107-117.

30. Pascoa LA, Elzo A, Ferreira DL, Bezerra LA, Barbosa R. Effect of several structures of (co) variances and genetic parameters for weaning weight in Nellore cattle. Ciênc Anim Bras 2013;(14):151-158.

31. Oliveira JGA, Pereira J, Ramírez-Díaz J, Sterman JB, Santana ML. Inclusion of weaning management group as a random effect in the genetic evaluation of postweaning traits in Nellore. Trop Anim Health Prod 2014;(46):1031-1036.

32. Pedrosa VB, Eler JP, Ferraz JBS, Groeneveld E. Use of weaning management group as a random effect for a more robust estimation of genetic parameters for post-weaning traits in Nellore cattle. Genet Mol Res 2014;13(3):7013-7021.

33. Saavedra-Jiménez LA, Ramírez-Valverde R, Núñez-Domínguez R, García-Muñiz J, Lopez-Villalobos N, Ruiz-Flores A. Genotype by climate interaction in the genetic evaluation for growing traits of Braunvieh cattle in Mexico. Trop Anim Health Prod 2013;45(7):1489-1494. 
34. Estrada León RJ, Magaña JG, Segura JC. Comparación de modelos en la evaluación genética de caracteres de crecimiento del ganado Brahman en el sureste de México. Arch Latinoam Prod Anim 2008;(16):224-233.

35. Domínguez-Viveros J, Rodríguez-Almeida FA, Núñez-Domínguez R, OrtegaGutiérrez JA, Ramírez-Valverde R; Santellano-Estrada E, Espinosa-Villavicencio JL. Ajuste de modelos de regresión aleatoria en evaluaciones genéticas de bovinos tropicarne. Agrociencia 2011;(45):325-337.

36. Córdova RA, Rodríguez G, Córdova M, Córdova C, Pérez J. Ganancia diaria y peso al destete en terneros de cruces de Bos taurus y Bos indicus en trópico húmedo. Rev MVZ Córdoba 2005;(10):589-592.

37. Hargraves GH. Defining and using reference evapotranspiration. J Irrigation Drainage Eng 1994;(20):1132-1139.

\section{Anexo 1: Clasificación de épocas climáticas en diferentes municipios de México} usando el índice de aridez

\begin{tabular}{|c|c|c|c|c|c|c|c|c|c|c|c|c|c|}
\hline Estado & Municipio & 1 & 2 & 3 & 4 & 5 & 6 & 7 & 8 & 9 & 10 & 11 & 12 \\
\hline Aguascalientes & Aguascalientes & SA & $A$ & $A$ & A & A & $\mathrm{SH}$ & $\mathrm{H}$ & $\mathrm{SH}$ & $\mathrm{H}$ & SA & A & A \\
\hline Aguascalientes & Calvillo & SA & $A$ & $A$ & $A$ & $A$ & $\mathrm{SH}$ & $\mathrm{H}$ & $\mathrm{H}$ & $\mathrm{H}$ & SA & $A$ & $A$ \\
\hline Aguascalientes & $\begin{array}{l}\text { Rincón de } \\
\text { Romos }\end{array}$ & SA & $A$ & $A$ & A & $A$ & SA & $\mathrm{H}$ & $\mathrm{SH}$ & $\mathrm{SH}$ & SA & A & $A$ \\
\hline Aguascalientes & Jesús María & SA & A & $A$ & A & A & $\mathrm{SH}$ & $\mathrm{H}$ & $\mathrm{H}$ & $\mathrm{SH}$ & SA & A & $A$ \\
\hline Baja California & Mexicali & $A$ & $A$ & $A$ & $A$ & $A$ & A & $A$ & $A$ & $A$ & A & A & SA \\
\hline Baja California & Ensenada & $\mathrm{SH}$ & $A$ & $A$ & A & A & A & $A$ & A & $A$ & A & A & $A$ \\
\hline Baja California & Tijuana & $\mathrm{H}$ & $\mathrm{SH}$ & $\mathrm{SH}$ & $A$ & A & $A$ & $A$ & $A$ & $A$ & A & $\mathrm{SH}$ & $\mathrm{H}$ \\
\hline BC Sur & La Paz & SA & $A$ & $A$ & A & A & A & $A$ & SA & SA & A & $A$ & SA \\
\hline BC Sur & Mulege & $A$ & A & $A$ & A & $A$ & A & $A$ & $A$ & SA & $A$ & $A$ & $A$ \\
\hline Campeche & Campeche & SA & $A$ & $A$ & $A$ & SA & $\mathrm{H}$ & $\mathrm{H}$ & $\mathrm{H}$ & $\mathrm{H}$ & $\mathrm{H}$ & $\mathrm{SH}$ & SA \\
\hline Campeche & Escárcega & $\mathrm{SH}$ & SA & $A$ & $A$ & $\mathrm{SH}$ & $\mathrm{H}$ & $\mathrm{H}$ & $\mathrm{H}$ & $\mathrm{H}$ & $\mathrm{H}$ & $\mathrm{H}$ & $\mathrm{SH}$ \\
\hline Campeche & Calkiní & SA & SA & $A$ & SA & SA & $\mathrm{H}$ & $\mathrm{H}$ & $\mathrm{H}$ & $\mathrm{H}$ & $\mathrm{H}$ & $\mathrm{H}$ & $\mathrm{SH}$ \\
\hline Campeche & Champotón & SA & SA & $A$ & $A$ & SA & $\mathrm{H}$ & $\mathrm{H}$ & $\mathrm{H}$ & $\mathrm{H}$ & $\mathrm{H}$ & $\mathrm{H}$ & $\mathrm{SH}$ \\
\hline Chiapas & Tapachula & $\mathrm{SH}$ & $\mathrm{SH}$ & $A$ & $\mathrm{SH}$ & $\mathrm{H}$ & $\mathrm{H}$ & $\mathrm{H}$ & $\mathrm{H}$ & $\mathrm{H}$ & $\mathrm{H}$ & $\mathrm{H}$ & $A$ \\
\hline Chiapas & Tuxtla Gutiérrez & $A$ & $A$ & $A$ & $A$ & SA & $\mathrm{H}$ & $\mathrm{H}$ & $\mathrm{H}$ & $\mathrm{H}$ & $\mathrm{H}$ & $A$ & $A$ \\
\hline Chiapas & Palenque & $\mathrm{H}$ & $\mathrm{H}$ & SA & SA & $\mathrm{H}$ & $\mathrm{H}$ & $\mathrm{H}$ & $\mathrm{H}$ & $\mathrm{H}$ & $\mathrm{H}$ & $\mathrm{H}$ & $\mathrm{H}$ \\
\hline Chiapas & Comitán & A & $A$ & $A$ & SA & $\mathrm{H}$ & $\mathrm{H}$ & $\mathrm{H}$ & $\mathrm{H}$ & $\mathrm{H}$ & $\mathrm{H}$ & SA & $A$ \\
\hline Chihuahua & Buenaventura & $A$ & $A$ & $A$ & $A$ & $A$ & SA & $\mathrm{H}$ & $\mathrm{H}$ & $\mathrm{H}$ & SA & $A$ & $A$ \\
\hline Chihuahua & Delicias & $A$ & A & $A$ & $A$ & $A$ & A & SA & SA & SA & $A$ & $A$ & $A$ \\
\hline
\end{tabular}




\begin{tabular}{|c|c|c|c|c|c|c|c|c|c|c|c|c|c|}
\hline Chihuahua & Ahumada & A & $A$ & $A$ & A & $A$ & $A$ & SA & SA & SA & SA & A & SA \\
\hline Chihuahua & Cuauhtémoc & $A$ & $A$ & A & SA & A & SA & $\mathrm{H}$ & $\mathrm{H}$ & $\mathrm{H}$ & SA & A & A \\
\hline Coahuila & Torreón & $A$ & $A$ & $A$ & $A$ & $A$ & $A$ & SA & SA & SA & $A$ & $A$ & $A$ \\
\hline Coahuila & Sabinas & $A$ & A & A & SA & SA & SA & A & SA & $\mathrm{SH}$ & $\mathrm{SH}$ & SA & SA \\
\hline Coahuila & Matamoros & $A$ & $A$ & $A$ & $A$ & $A$ & $A$ & $A$ & SA & $A$ & $A$ & $A$ & $A$ \\
\hline Coahuila & Saltillo & SA & $A$ & $A$ & A & $A$ & SA & SA & SA & $\mathrm{SH}$ & SA & A & $A$ \\
\hline Colima & Cuauhtémoc & SA & $A$ & $A$ & $A$ & $A$ & $\mathrm{H}$ & $\mathrm{H}$ & $\mathrm{H}$ & $\mathrm{H}$ & $\mathrm{H}$ & SA & $A$ \\
\hline Colima & Tecomán & SA & A & A & $A$ & A & $\mathrm{H}$ & $\mathrm{H}$ & $\mathrm{H}$ & $\mathrm{H}$ & $\mathrm{H}$ & SA & $A$ \\
\hline Durango & Mezquite & SA & $A$ & $A$ & $A$ & $A$ & SA & $\mathrm{H}$ & $\mathrm{H}$ & $\mathrm{SH}$ & $\mathrm{SH}$ & $A$ & SA \\
\hline Durango & Gómez Palacio & $A$ & $A$ & A & $A$ & A & SA & A & SA & SA & $A$ & $A$ & $A$ \\
\hline Durango & Tamazula & SA & A & A & $A$ & A & SA & $\mathrm{H}$ & $\mathrm{H}$ & $\mathrm{H}$ & $\mathrm{SH}$ & SA & $\mathrm{SH}$ \\
\hline Durango & Tlahualilo & $A$ & A & A & $A$ & $A$ & SA & SA & SA & SA & $A$ & $A$ & $A$ \\
\hline Durango & Cuencamé & A & $A$ & A & $A$ & A & SA & SA & $\mathrm{SH}$ & $\mathrm{SH}$ & SA & A & A \\
\hline Edo. de México & Temascaltepec & $A$ & $A$ & $A$ & $A$ & SA & $\mathrm{H}$ & $\mathrm{H}$ & $\mathrm{H}$ & $\mathrm{H}$ & $\mathrm{H}$ & SA & $A$ \\
\hline Edo. de México & Toluca & $A$ & $A$ & $A$ & SA & $\mathrm{SH}$ & $\mathrm{H}$ & $\mathrm{H}$ & $\mathrm{H}$ & $\mathrm{H}$ & $\mathrm{SH}$ & $A$ & $A$ \\
\hline Guanajuato & Celaya & $A$ & $A$ & A & $A$ & A & $\mathrm{H}$ & $\mathrm{H}$ & $\mathrm{H}$ & $\mathrm{H}$ & SA & A & A \\
\hline Guanajuato & León & $A$ & $A$ & A & $A$ & $A$ & $\mathrm{H}$ & $\mathrm{H}$ & $\mathrm{H}$ & $\mathrm{H}$ & SA & A & A \\
\hline Guanajuato & SL de La Paz & $A$ & $A$ & $A$ & $A$ & SA & $\mathrm{SH}$ & $\mathrm{SH}$ & SA & $\mathrm{H}$ & SA & $A$ & $A$ \\
\hline Guanajuato & Abasolo & $A$ & $A$ & A & $A$ & $A$ & $\mathrm{H}$ & $\mathrm{H}$ & $\mathrm{H}$ & $\mathrm{H}$ & SA & A & A \\
\hline Guerrero & Chilpancingo & A & A & A & $A$ & SA & $\mathrm{H}$ & $\mathrm{H}$ & $\mathrm{H}$ & $\mathrm{H}$ & $\mathrm{H}$ & A & A \\
\hline Guerrero & Cuajinicuilapa & $A$ & A & A & $A$ & SA & $\mathrm{H}$ & $\mathrm{H}$ & $\mathrm{H}$ & $\mathrm{H}$ & $\mathrm{H}$ & $A$ & A \\
\hline Guerrero & Iguala & $A$ & $A$ & $A$ & $A$ & SA & $\mathrm{H}$ & $\mathrm{H}$ & $\mathrm{H}$ & $\mathrm{H}$ & $\mathrm{H}$ & $A$ & $A$ \\
\hline Hidalgo & Huejutla & $\mathrm{H}$ & $\mathrm{SH}$ & SA & SA & $\mathrm{SH}$ & $\mathrm{H}$ & $\mathrm{H}$ & $\mathrm{H}$ & $\mathrm{H}$ & $\mathrm{H}$ & $\mathrm{H}$ & $\mathrm{H}$ \\
\hline Hidalgo & Zacualtipán & $\mathrm{SH}$ & SA & SA & SA & SA & $\mathrm{H}$ & $\mathrm{H}$ & $\mathrm{H}$ & $\mathrm{H}$ & $\mathrm{H}$ & $\mathrm{H}$ & $\mathrm{SH}$ \\
\hline Hidalgo & Pachuca & $A$ & A & $A$ & $A$ & SA & $\mathrm{SH}$ & $\mathrm{SH}$ & SA & $\mathrm{SH}$ & SA & A & $A$ \\
\hline Hidalgo & Actopan & $A$ & $A$ & $A$ & $A$ & SA & SA & SA & SA & $\mathrm{SH}$ & SA & $A$ & $A$ \\
\hline Jalisco & Tonalá & A & $A$ & A & $A$ & $\mathrm{H}$ & $\mathrm{H}$ & $\mathrm{H}$ & $\mathrm{H}$ & $\mathrm{H}$ & SA & $A$ & A \\
\hline Jalisco & Arandas & $A$ & $A$ & A & $A$ & SA & $\mathrm{H}$ & $\mathrm{H}$ & $\mathrm{H}$ & $\mathrm{H}$ & SA & SA & $A$ \\
\hline Jalisco & Lagos de Moreno & $A$ & $A$ & $A$ & $A$ & $A$ & $\mathrm{H}$ & $\mathrm{H}$ & $\mathrm{H}$ & $\mathrm{H}$ & SA & $A$ & SA \\
\hline Jalisco & Ameca & SA & A & A & $A$ & $A$ & $\mathrm{H}$ & $\mathrm{H}$ & $\mathrm{H}$ & $\mathrm{H}$ & SA & A & $A$ \\
\hline Jalisco & Tepatitlán & $A$ & A & A & $A$ & SA & $\mathrm{H}$ & $\mathrm{H}$ & $\mathrm{H}$ & $\mathrm{H}$ & SA & A & A \\
\hline Jalisco & Cd. Guzmán & SA & A & A & $A$ & $A$ & $\mathrm{H}$ & $\mathrm{H}$ & $\mathrm{H}$ & $\mathrm{H}$ & SA & $A$ & A \\
\hline Michoacán & Arteaga & SA & A & A & $A$ & A & $\mathrm{H}$ & $\mathrm{H}$ & $\mathrm{H}$ & $\mathrm{H}$ & $\mathrm{H}$ & SA & $A$ \\
\hline Michoacán & Apatzingán & $A$ & A & A & $A$ & A & $\mathrm{H}$ & $\mathrm{H}$ & $\mathrm{H}$ & $\mathrm{H}$ & $\mathrm{SH}$ & $A$ & $A$ \\
\hline Michoacán & La Huacana & A & A & A & $A$ & $A$ & $\mathrm{H}$ & $\mathrm{H}$ & $\mathrm{H}$ & $\mathrm{H}$ & $\mathrm{H}$ & A & A \\
\hline Michoacán & Sahuayo & SA & A & A & $A$ & A & $\mathrm{H}$ & $\mathrm{H}$ & $\mathrm{H}$ & $\mathrm{H}$ & SA & A & A \\
\hline Morelos & Cuautla & $A$ & $A$ & A & $A$ & SA & $\mathrm{H}$ & $\mathrm{H}$ & $\mathrm{H}$ & $\mathrm{H}$ & $\mathrm{SH}$ & $A$ & $A$ \\
\hline Morelos & Tepalcingo & A & $A$ & A & $A$ & SA & $\mathrm{H}$ & $\mathrm{H}$ & $\mathrm{H}$ & $\mathrm{H}$ & $\mathrm{SH}$ & $A$ & $A$ \\
\hline Nayarit & $\begin{array}{l}\text { Santiago } \\
\text { Ixcuintla }\end{array}$ & SA & A & A & $A$ & $A$ & $\mathrm{H}$ & $\mathrm{H}$ & $\mathrm{H}$ & $\mathrm{H}$ & $\mathrm{H}$ & SA & SA \\
\hline Nayarit & La Yesca & A & $A$ & A & $A$ & $A$ & $\mathrm{H}$ & $\mathrm{H}$ & $\mathrm{H}$ & $\mathrm{H}$ & SA & $A$ & $A$ \\
\hline Nayarit & Compostela & $A$ & $A$ & $A$ & $A$ & $A$ & $\mathrm{H}$ & $\mathrm{H}$ & $\mathrm{H}$ & $\mathrm{H}$ & $\mathrm{SH}$ & A & SA \\
\hline
\end{tabular}




\begin{tabular}{|c|c|c|c|c|c|c|c|c|c|c|c|c|c|}
\hline Nayarit & Tepic & SA & SA & A & A & $A$ & $\mathrm{H}$ & $\mathrm{H}$ & $\mathrm{H}$ & $\mathrm{H}$ & $\mathrm{H}$ & SA & $\mathrm{SH}$ \\
\hline Nuevo León & Galeana & $A$ & $A$ & $A$ & $A$ & SA & SA & SA & SA & $\mathrm{H}$ & SA & A & A \\
\hline Nuevo León & Villaldama & SA & $A$ & $A$ & $A$ & SA & SA & SA & SA & SA & SA & SA & SA \\
\hline Nuevo León & Monterrey & SA & SA & SA & SA & $\mathrm{SH}$ & $\mathrm{SH}$ & SA & $\mathrm{SH}$ & $\mathrm{H}$ & $\mathrm{H}$ & SA & SA \\
\hline Nuevo León & Apodaca & SA & SA & $A$ & SA & SA & SA & SA & SA & $\mathrm{H}$ & $\mathrm{SH}$ & SA & SA \\
\hline Oaxaca & Tuxtepec & $\mathrm{H}$ & SA & SA & SA & $\mathrm{SH}$ & $\mathrm{H}$ & $\mathrm{H}$ & $\mathrm{H}$ & $\mathrm{H}$ & $\mathrm{H}$ & $\mathrm{H}$ & $\mathrm{SH}$ \\
\hline Oaxaca & $\begin{array}{l}\text { San Pedro } \\
\text { Mixtepec }\end{array}$ & A & $A$ & A & A & SA & $\mathrm{H}$ & $\mathrm{H}$ & $\mathrm{H}$ & $\mathrm{H}$ & $\mathrm{SH}$ & A & A \\
\hline Oaxaca & $\begin{array}{l}\text { Pinotepa } \\
\text { Nacional }\end{array}$ & A & $A$ & A & A & A & $\mathrm{H}$ & $\mathrm{H}$ & $\mathrm{H}$ & $\mathrm{H}$ & $\mathrm{H}$ & A & $A$ \\
\hline Oaxaca & Juchitán & $A$ & $A$ & A & $A$ & SA & $\mathrm{H}$ & $\mathrm{H}$ & $\mathrm{H}$ & $\mathrm{H}$ & $\mathrm{H}$ & SA & $A$ \\
\hline Puebla & Tehuacán & $A$ & $A$ & $A$ & $A$ & SA & $\mathrm{H}$ & $\mathrm{SH}$ & $\mathrm{SH}$ & $\mathrm{H}$ & SA & $A$ & $A$ \\
\hline Puebla & Tlacotepec & $A$ & A & A & SA & $\mathrm{SH}$ & $\mathrm{H}$ & SA & SA & $\mathrm{H}$ & SA & A & A \\
\hline Puebla & Tecamachalco & $A$ & $A$ & $A$ & SA & $\mathrm{SH}$ & $\mathrm{H}$ & $\mathrm{SH}$ & $\mathrm{SH}$ & $\mathrm{H}$ & SA & A & $A$ \\
\hline Q. Roo & Chetumal & $\mathrm{H}$ & SA & SA & SA & $\mathrm{H}$ & $\mathrm{H}$ & $\mathrm{H}$ & $\mathrm{H}$ & $\mathrm{H}$ & $\mathrm{H}$ & $\mathrm{H}$ & $\mathrm{H}$ \\
\hline Q. Roo & Felipe Carrillo P. & $\mathrm{H}$ & $\mathrm{SH}$ & SA & SA & $\mathrm{H}$ & $\mathrm{H}$ & $\mathrm{H}$ & $\mathrm{H}$ & $\mathrm{H}$ & $\mathrm{H}$ & $\mathrm{H}$ & $\mathrm{H}$ \\
\hline Q. Roo & Bacalar & $\mathrm{SH}$ & $\mathrm{SH}$ & SA & SA & SA & $\mathrm{H}$ & $\mathrm{H}$ & $\mathrm{H}$ & $\mathrm{H}$ & $\mathrm{H}$ & $\mathrm{H}$ & $\mathrm{H}$ \\
\hline Querétaro & San Juan del Río & $A$ & $A$ & $A$ & $A$ & SA & $\mathrm{H}$ & $\mathrm{H}$ & $\mathrm{H}$ & $\mathrm{H}$ & $\mathrm{SH}$ & A & A \\
\hline Querétaro & Ezequiel Montes & A & $A$ & A & $A$ & $\mathrm{SH}$ & SA & $\mathrm{H}$ & SA & SA & SA & A & $A$ \\
\hline Querétaro & Querétaro & $A$ & SA & A & A & SA & $\mathrm{SH}$ & $\mathrm{H}$ & $\mathrm{SH}$ & $\mathrm{H}$ & SA & $A$ & A \\
\hline Sinaloa & Guasave & SA & $A$ & A & A & $A$ & $A$ & $\mathrm{SH}$ & $\mathrm{H}$ & $\mathrm{H}$ & SA & SA & SA \\
\hline Sinaloa & Culiacán & SA & $A$ & A & A & $A$ & A & $\mathrm{H}$ & $\mathrm{H}$ & $\mathrm{H}$ & SA & SA & SA \\
\hline Sinaloa & Mocorito & SA & $A$ & A & A & A & A & $\mathrm{H}$ & $\mathrm{H}$ & $\mathrm{H}$ & SA & SA & SA \\
\hline Sinaloa & Mazatlán & $A$ & A & A & A & $A$ & SA & $\mathrm{H}$ & $\mathrm{H}$ & $\mathrm{H}$ & $A$ & A & A \\
\hline San Luis Potosí & San Luis Potosí & $A$ & A & A & A & SA & SA & $\mathrm{SH}$ & SA & $\mathrm{SH}$ & SA & A & A \\
\hline San Luis Potosí & Villa de Arriaga & $A$ & A & A & $A$ & SA & SA & $\mathrm{SH}$ & SA & $\mathrm{SH}$ & SA & A & $A$ \\
\hline San Luis Potosí & Matehuala & SA & A & A & SA & SA & $\mathrm{SH}$ & $\mathrm{SH}$ & $\mathrm{SH}$ & $\mathrm{H}$ & SA & A & SA \\
\hline San Luis Potosí & Ciudad del Maíz & $A$ & $A$ & A & $A$ & SA & SA & $\mathrm{H}$ & $\mathrm{SH}$ & $\mathrm{H}$ & $\mathrm{SH}$ & SA & SA \\
\hline Sonora & Agua Prieta & SA & SA & A & A & $A$ & $A$ & $\mathrm{SH}$ & $\mathrm{SH}$ & SA & SA & SA & $\mathrm{SH}$ \\
\hline Sonora & Ures & SA & SA & A & A & A & A & $\mathrm{SH}$ & $\mathrm{SH}$ & $\mathrm{SH}$ & $A$ & $A$ & $\mathrm{SH}$ \\
\hline Sonora & Rosario & $A$ & $A$ & A & A & A & SA & $\mathrm{H}$ & $\mathrm{H}$ & $\mathrm{H}$ & $A$ & A & $A$ \\
\hline Sonora & Álamos & SA & SA & A & A & A & $A$ & $\mathrm{H}$ & $\mathrm{H}$ & $\mathrm{H}$ & SA & SA & SA \\
\hline Sonora & Navojoa & SA & $A$ & $A$ & $A$ & $A$ & A & $\mathrm{SH}$ & $\mathrm{H}$ & $\mathrm{H}$ & SA & $A$ & SA \\
\hline Tabasco & Villahermosa & $\mathrm{H}$ & $\mathrm{H}$ & SA & SA & $\mathrm{SH}$ & $\mathrm{H}$ & $\mathrm{H}$ & $\mathrm{H}$ & $\mathrm{H}$ & $\mathrm{H}$ & $\mathrm{H}$ & $\mathrm{H}$ \\
\hline Tabasco & Balancán & $\mathrm{H}$ & $\mathrm{SH}$ & SA & SA & $\mathrm{H}$ & $\mathrm{H}$ & $\mathrm{H}$ & $\mathrm{H}$ & $\mathrm{H}$ & $\mathrm{H}$ & $\mathrm{H}$ & $\mathrm{H}$ \\
\hline Tabasco & Jonuta & $\mathrm{H}$ & $\mathrm{H}$ & SA & SA & $\mathrm{SH}$ & $\mathrm{H}$ & $\mathrm{H}$ & $\mathrm{H}$ & $\mathrm{H}$ & $\mathrm{H}$ & $\mathrm{H}$ & $\mathrm{H}$ \\
\hline Tabasco & Jalpa de Méndez & $\mathrm{H}$ & $\mathrm{H}$ & SA & SA & SA & $\mathrm{H}$ & $\mathrm{H}$ & $\mathrm{H}$ & $\mathrm{H}$ & $\mathrm{H}$ & $\mathrm{H}$ & $\mathrm{H}$ \\
\hline Tamaulipas & Aldama & SA & $A$ & $A$ & $A$ & SA & $\mathrm{H}$ & $\mathrm{H}$ & $\mathrm{H}$ & $\mathrm{H}$ & $\mathrm{H}$ & SA & SA \\
\hline Tamaulipas & Tampico & SA & $\mathrm{SH}$ & $A$ & $A$ & SA & $\mathrm{H}$ & $\mathrm{H}$ & $\mathrm{H}$ & $\mathrm{H}$ & $\mathrm{H}$ & $A$ & SA \\
\hline Tamaulipas & Victoria & SA & SA & $A$ & SA & $\mathrm{SH}$ & $\mathrm{H}$ & SA & $\mathrm{H}$ & $\mathrm{H}$ & $\mathrm{H}$ & SA & A \\
\hline Tamaulipas & Nuevo Laredo & SA & SA & $A$ & SA & SA & SA & $A$ & SA & $\mathrm{SH}$ & $\mathrm{H}$ & SA & SA \\
\hline Veracruz & Acayucan & $\mathrm{SH}$ & SA & $A$ & $A$ & SA & $\mathrm{H}$ & $\mathrm{H}$ & $\mathrm{H}$ & $\mathrm{H}$ & $\mathrm{H}$ & $\mathrm{H}$ & $\mathrm{H}$ \\
\hline
\end{tabular}


Revista Mexicana de Ciencias Pecuarias Volumen 9 Número 42018

\begin{tabular}{llllllllllllll} 
Veracruz & Veracruz & SA & SA & H & A & SA & H & H & H & H & SH & H & SA \\
Veracruz & Minatitlan & H & H & SA & SA & H & H & H & H & H & H & H & H \\
Veracruz & Ozuluama & H & SA & A & SA & SA & H & H & H & H & H & H & H \\
Yucatán & Mérida & SH & SA & SA & SA & SH & H & H & H & H & H & H & SH \\
Yucatán & Tizimín & SH & SA & SA & SA & H & H & H & H & H & H & SH & H \\
Yucatán & Bucztotz & SH & SA & SA & A & SH & H & H & H & H & H & SH & SH \\
Yucatán & Oxkutzcab & SA & SA & SA & SA & SH & H & H & H & H & H & SH & H \\
\hline
\end{tabular}

1-12= meses del año, $\mathrm{A}=$ árido, $\mathrm{SA}=$ semiárido, $\mathrm{SH}=$ subhúmedo $\mathrm{H}=$ húmedo. 